\title{
Towards a differential equation for the nonrelativistic ground-state electron density of the He-like sequence of atomic ions
}

\author{
I. A. Howard ${ }^{1}$ and N. H. March ${ }^{1,2}$ \\ ${ }^{1}$ Department of Physics, University of Antwerp, Groenenborgerlaan 171, B-2020 Antwerp, Belgium \\ ${ }^{2}$ Oxford University, Oxford, England \\ (Received 20 December 2004; published 1 April 2005)
}

\begin{abstract}
The early study of Schwartz [Ann. Phys. (N.Y.) 6, 156 (1959)] led to an explicit expression for the ground-state electron density $\rho(r)$ of He-like atomic ions with nuclear charge Ze in the limit of large $Z$. Much later, Gál, March, and Nagy [Chem. Phys. Lett. 305, 429 (1999)] derived a third-order linear homogeneous differential equation satisfied by the Schwartz limiting density. Our aim here has been to solve a still correlated model more closely related to the He atom itself. Motivated by semiclassical studies (as by Handke [Phys. Rev. A 50, R3561 (1994)]), we have solved a fully quantal model in which the Coulomb repulsion energy $e^{2} / r_{12}$ of the two electrons at separation $r_{12}$ is expanded in a one-center form about the atomic nucleus and only the $s$-wave term is retained. From the resulting analytical ground-state density, a differential equation has been derived, which is contrasted with that satisfied by the large- $Z$ limiting form of Schwartz. Suggestions are finally made as to the way the density of the He atomic ion series may be characterized, the ionization potential $I(Z)$ being proposed as crucial input data.
\end{abstract}

DOI: 10.1103/PhysRevA.71.042501

PACS number(s): $31.10 .+\mathrm{z}$

\section{BACKGROUND AND OUTLINE}

Since the advent of quantum mechanics some eight decades ago, multifarious attempts have been made to solve the Schrödinger equation for the ground state of the He atom. To date, however, an exact solution has not been discovered. Therefore, it is relevant background to the present analytical considerations to note that, in semiclassical studies of the above problem, some attention has been directed towards a simplified Hamiltonian, as exemplified by the work of Handke [1] (see also earlier references cited there). In this Hamiltonian [see Eq. (2.1) below for its explicit form] the electron-electron Coulombic repulsion energy $e^{2} / r_{12}$ between the two (spin-compensated) electrons at separation $r_{12}$ is approximated by the lowest-order ( $s$-wave-like) term in a "one-center" expansion.

What is demonstrated here is that the resulting simplified Hamiltonian is amenable to exact solution in terms of hydrogenic functions. This solution is set out for the symmetric spatial Schrödinger wave function $\Psi\left(\mathbf{r}_{1}, \mathbf{r}_{2}\right)$ in Sec. II below. From this, the ground-state electron density is derived. Section III is concerned with the differential equation satisfied by this density. The limiting Schwartz form yields a thirdorder linear homogeneous differential equation derived by Gál, March, and Nagy [2], and in Sec. IV the equation is compared and contrasted with the new differential equation derived in the present study. Section V constitutes a summary plus some suggestions for future work.

\section{He $s$-WAVE HAMILTONIAN: EXACT ANALYTICAL SOLUTION FOR GROUND-STATE ELECTRON DENSITY}

The Schrödinger equation of this model has the explicit form [1] (atomic units are used throughout)

$$
\left[-\frac{\nabla_{1}^{2}}{2}-\frac{\nabla_{2}^{2}}{2}-\frac{Z}{r_{1}}-\frac{Z}{r_{2}}+\frac{1}{r_{>}}\right] \Psi\left(r_{1}, r_{2}\right)=E \Psi\left(r_{1}, r_{2}\right) .
$$

Thus, as an example, for $r_{1}>r_{2}$ we have

$$
\left[-\frac{\nabla_{1}^{2}}{2}-\frac{\nabla_{2}^{2}}{2}-\frac{Z}{r_{1}}-\frac{Z}{r_{2}}+\frac{1}{r_{1}}\right] \Psi\left(r_{1}, r_{2}\right)=E \Psi\left(r_{1}, r_{2}\right) .
$$

Explicitly, this has the separable structure

$$
\left\{\left[-\frac{\nabla_{1}^{2}}{2}-\frac{(Z-1)}{r_{1}}\right]+\left[\frac{\nabla_{2}^{2}}{2}-\frac{Z}{r_{2}}\right]\right\} \Psi\left(r_{1}, r_{2}\right)=E \Psi\left(r_{1}, r_{2}\right),
$$

so the solution is of the form

$$
\Psi\left(r_{1}, r_{2}\right)=\varphi_{0 n}\left(Z-1, r_{1}\right) \varphi_{0 m}\left(Z, r_{2}\right),
$$

where $\varphi_{0 n}(Z, r)$ is an $l=0$ solution for the hydrogen atom. The energy $E$ is then

$$
E=-\frac{(Z-1)^{2}}{2 n^{2}}-\frac{Z^{2}}{2 m^{2}}
$$

For $r_{2}>r_{1}$ we have then, of course,

$$
\Psi\left(r_{1}, r_{2}\right)=\varphi_{0 m}\left(Z, r_{1}\right) \varphi_{0 n}\left(Z-1, r_{2}\right) .
$$

Therefore the ground-state solution (symmetric and not normalized) is 


$$
\begin{aligned}
\Psi\left(r_{1}, r_{2}\right)= & {\left[\frac{(Z-1)^{3}}{\pi}\right]^{1 / 2} \exp \left[-(Z-1) r_{1}\right]\left[\frac{Z^{3}}{\pi}\right]^{1 / 2} } \\
& \times \exp \left(-Z r_{2}\right) \quad\left(r_{1}>r_{2}\right) \\
& =\left[\frac{Z^{3}}{\pi}\right]^{1 / 2} \exp \left(-Z r_{1}\right)\left[\frac{(Z-1)^{3}}{\pi}\right]^{1 / 2} \\
& \times \exp \left[-(Z-1) r_{2}\right]\left(r_{2}>r_{1}\right),
\end{aligned}
$$

It follows that the ground-state density can be written

with energy

$$
\begin{aligned}
\rho\left(r_{1}\right)= & \int \Psi^{2}\left(r_{1}, r_{2}\right) d \mathbf{r}_{2} \\
= & \varphi_{01}^{2}\left(Z-1, r_{1}\right) \int_{r 2=0}^{r 1} 4 \pi r_{2}^{2} \varphi_{01}^{2}\left(Z, r_{2}\right) d r_{2} \\
& +\varphi_{01}^{2}\left(Z, r_{1}\right) \int_{r 2=r 1}^{\infty} 4 \pi r_{2}^{2} \varphi_{01}^{2}\left(Z-1, r_{2}\right) d r_{2}
\end{aligned}
$$

$$
E=-\frac{Z^{2}}{2}-\frac{(Z-1)^{2}}{2}=-\left(Z^{2}+\frac{1}{2}\right)+Z
$$

or

$$
\rho\left(r_{1}\right)=\frac{(Z-1)^{3}}{\pi} \exp \left[-2(Z-1) r_{1}\right] \frac{Z^{3}}{\pi} 4 \pi \int_{r 2=0}^{r 1} r_{2}^{2} \exp \left(-2 Z r_{2}\right) d r_{2}+\frac{Z^{3}}{\pi} \exp \left(-2 Z r_{1}\right) \frac{(Z-1)^{3}}{\pi} 4 \pi \int_{r 2=r 1}^{\infty} r_{2}^{2} \exp \left[-2(Z-1) r_{2}\right] d r_{2}
$$

Explicitly, the ground-state density (unnormalized) is therefore of the form

$$
\begin{aligned}
\rho\left(r_{1}\right)= & \frac{(Z-1)^{3}}{\pi} \exp \left[-2(Z-1) r_{1}\right]\left[1-\left(1+2 Z r_{1}+2 Z^{2} r_{1}^{2}\right) \exp \left(-2 Z r_{1}\right)\right]+\frac{Z^{3}}{\pi} \exp \left(-2 Z r_{1}\right) \exp \left[-2(Z-1) r_{1}\right] \\
& \times\left[1+2(Z-1) r_{1}+2(Z-1)^{2} r_{1}^{2}\right] .
\end{aligned}
$$

\section{A. Verification of Kato's theorem}

One must have, at the nucleus, the electron-nuclear cusp condition [3]

$$
\left.\frac{\partial \rho(r)}{\partial r}\right|_{r=0}=-2 Z \rho(r=0) .
$$

From the (as-yet unnormalized) form (2.11), we find almost immediately that

$$
\rho(r=0)=\frac{Z^{3}}{\pi} .
$$

Since we shall require the result below when we derive the differential equation satisfied by $\rho(r)$ in Eq. (2.11), let us write the derivative $\partial \rho(r) / \partial r$ explicitly as

$$
\begin{aligned}
\rho^{\prime}(r)= & -2(Z-1) A_{0} \exp [-2(Z-1) r] \\
& -(4 Z-2)\left[A_{1}+A_{2} r+A_{3} r^{2}\right] \exp [-(4 Z-2) r] \\
& +\left[A_{2}+2 A_{3} r\right] \exp [-(4 Z-2) r],
\end{aligned}
$$

where the coefficients $A_{i}$ are determined by rewriting Eq. (2.11) as

$$
\begin{aligned}
\rho(r)= & A_{0} \exp [-2(Z-1) r]+\left[A_{1}+A_{2} r+A_{3} r^{2}\right] \\
& \times \exp [-(4 Z-2) r] .
\end{aligned}
$$

Setting $r=0$ in Eq. (2.14) and using Eq. (2.13) plus the form of the coefficients $A_{i}$ given in Table $\mathrm{I}$, it is readily verified that Eq. (2.12) is obeyed.

\section{B. Normalization of $\rho(r)$ to two electrons}

We next note that

$$
C=\int_{0}^{\infty} 4 \pi r^{2} \rho(r) d r=\frac{2 Z^{3}\left(10+16 Z^{2}-25 Z\right)}{5\left(7 Z^{5}-16 Z^{4}+16 Z^{3}-8 Z^{2}+2 Z\right)-1},
$$

so $\rho(r)$ in Eq. (2.11) must be multiplied by $2 / C$ to normalize to two electrons.

TABLE I. Coefficients $A_{i}$ in the ground-state density $\rho(r)$ given in Eq. (2.15).

$$
\begin{gathered}
A_{0}=(Z-1)^{3} / \pi \\
A_{1}=\left[Z^{3}-(Z-1)^{3}\right] / \pi \\
A_{2}=2 Z(Z-1)(2 Z-1) / \pi \\
A_{3}=2 Z^{2}(Z-1)^{2} / \pi
\end{gathered}
$$




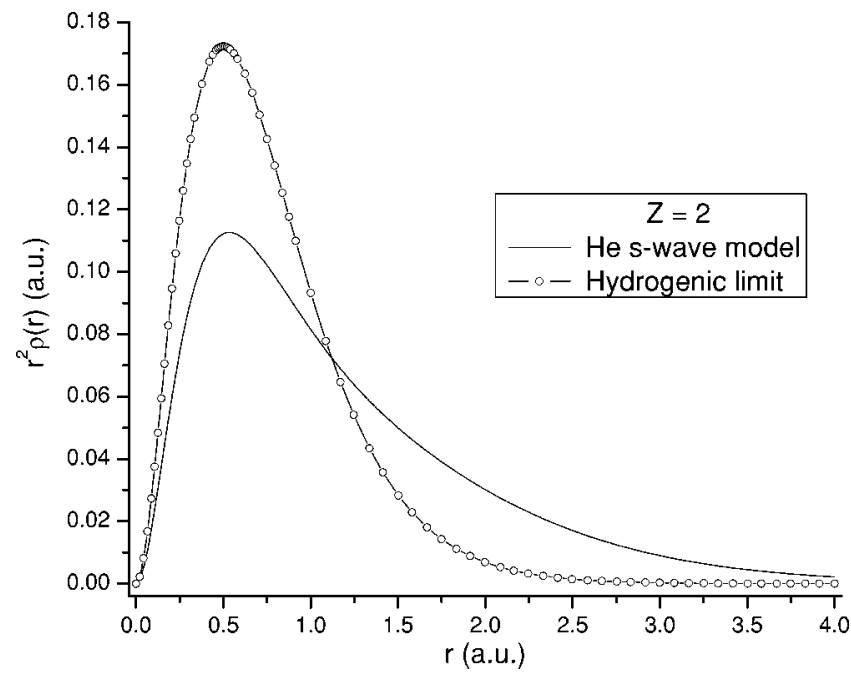

FIG. 1. Plot of the ground-state density $\rho(r)$ from Eq. (2.11) for $Z=2$. The hydrogenic density is shown for comparison, and the role of screening is seen to be crucial.

\section{Plots of $\rho(r)$ for $Z=2$ and $Z=92$}

Figures 1 and 2 show plots of $r^{2} \rho(r)$ versus $r$ in atomic units (a.u.) for $Z=2$ and $Z=92$, respectively. Of course, the $K$-shell electrons in the uranium atom move quite fast, but throughout we shall restrict ourselves entirely to properties which follow from the nonrelativistic Schrödinger equation. Figure 1 compares the "He $s$-wave" model ground-state density with the hydrogenic limit, both curves being normalized such that $\int_{0}^{\infty} 4 \pi r^{2} \rho(r) d r=2$. Obviously, screening plays a major role. In contrast, as $Z$ increases to 92, Figure 2 shows that the hydrogenic density is graphically the same as for the He-like sequence with averaged electron-electron interaction, as in Eq. (2.1).

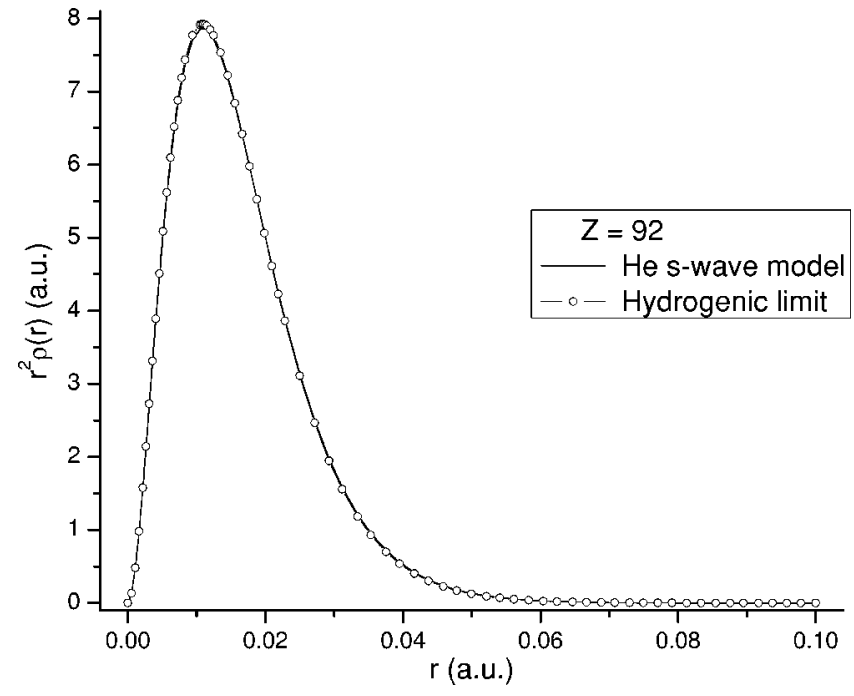

FIG. 2. Nonrelativistic ground-state density for He-like " $s$-wave" model with Eq. (2.11) used for $Z=92$. The result is now seen to be graphically indistinguishable from the hydrogenic limiting density shown.
TABLE II. Polynomials $P_{i s}(r)$ in second-order differential equation (4.2) for $\rho(r)$ of the He $s$-wave model. ${ }^{\mathrm{a}}$

$$
\begin{gathered}
P_{2 s}(r)=2 Z A_{3} r^{2}+2\left(Z A_{2}-A_{3}\right) r-A_{2} \\
P_{1 s}(r)=4 Z(3 Z-2) r^{2}+4\left[Z(3 Z-2) A_{2}-2(2 Z-1) A_{3}\right] r+4\left[Z(3 Z-2) A_{1}\right. \\
\left.-(2 Z-1) A_{2}\right] \\
P_{0 s}(r)=8 Z(2 Z-1)(Z-1) A_{3} r^{2}+4\left[-2(Z-1)(3 Z-1) A_{3}+2 Z(Z-1)\right. \\
\left.\times(2 Z-1) A_{2}\right] r \\
+4\left[2 Z(Z-1)(2 Z-1) A_{1}-(3 Z-1)(Z-1) A_{2}+(Z-1) A_{3}\right]
\end{gathered}
$$

${ }^{a}$ Note that coefficients $A_{i}$ are entered in earlier Table I.

\section{DIFFERENTIAL EQUATION FOR GROUND-STATE DENSITY $\rho(r)$}

The purpose of this section is to derive the differential equation satisfied by the exact ground-state density $\rho(r)$ given in Eq. (2.15) for the He $s$-wave model. One straightforward way to proceed is to multiply Eq. (2.15) throughout by $2(Z-1)$ and then to add the result to Eq. (2.14). One then removes from the sum thereby obtained the term $\exp [-2(Z$ -1)r] and with it the coefficient $A_{0}$. The explicit form then found is

$$
\begin{aligned}
\rho^{\prime}(r)+2(Z-1) \rho(r)= & {\left[\left(2 Z A_{1}+A_{2}\right)+2\left(Z A_{2}+A_{3}\right) r\right.} \\
& \left.+2 Z A_{3} r^{2}\right] \exp [-2(2 Z-1) r]
\end{aligned}
$$

Multiplying both sides of Eq. (3.1) by $\exp [2(2 Z-1) r]$ and differentiating once more then yields

$$
\begin{gathered}
\exp [2(2 Z-1) r]\left\{\left[\rho^{\prime \prime}(r)+2(Z-1) \rho^{\prime}(r)\right]+2(2 Z-1)\left[\rho^{\prime}(r)\right.\right. \\
+2(Z-1) \rho(r)]\}=\left[2\left(Z A_{2}+A_{3}\right)+4 Z A_{3} r\right] .
\end{gathered}
$$

Bringing the exponential factor in Eq. (3.2) back to the righthand side, this can then be removed from the resulting equation by employing Eq. (3.1). The outcome is a differential equation for $\rho$ of the form $\sum_{i=0}^{2} P_{i}(r) \rho^{(i)}(r)=0$ where the $P_{i}(r)$ denote polynomials which are given explicitly in Table II.

Equation (3.2) is a central finding of the present study and demonstrates that the ground-state density of the $\mathrm{He} s$-wave model satisfies a second-order linear homogeneous equation.

\section{RELATION TO THE LARGE-Z LIMIT OF DENSITY STUDIED BY SCHWARTZ}

Schwartz [4] calculated the limiting density for large $Z$, from Schrödinger's equation, for the He atomic ion sequence under discussion. Almost four decades later, Gál, March, and Nagy [2] derived the differential equation satisfied by $\rho(r)$ as given by Schwartz. They obtained

$$
P_{3 L}(r) \rho^{\prime \prime \prime}(r)+P_{2 L}(r) \rho^{\prime \prime}(r)+P_{1 L}(r) \rho^{\prime}(r)+P_{0 L}(r) \rho(r)=0,
$$

where the polynomials $P_{i L}(r)$ are recorded in Table III, $L$ denoting the limit of large atomic number $Z$.

Our objective in this section is now to compare and contrast the limiting result (4.1) for large $Z$ with the (second- 
TABLE III. Polynomials $P_{i L}(r)$ in Eq. (4.1) as derived by Gál $e t$ al. [2].

$$
\begin{gathered}
P_{3 L}(r)=-\left[9(Z r)^{2}+36(Z r)^{3}+40(Z r)^{4}\right] / Z^{3} \\
P_{2 L}(r)=-2\left[9(Z r)+54(Z r)^{2}+144(Z r)^{3}+160(Z r)^{4}\right] / Z^{2} \\
P_{1 L}(r)=2\left[9-138(Z r)^{2}-360(Z r)^{3}-400(Z r)^{4}\right] / Z \\
P_{0 L}(r)=4\left[9+18(Z r)-48(Z r)^{2}-144(Z r)^{3}-160(Z r)^{4}\right]
\end{gathered}
$$

order) differential equation (3.2) obtained here for the Heatomic-ion " $s$-wave" model. This result has the form

$$
P_{2 s}(r) \rho^{\prime \prime}(r)+P_{1 s}(r) \rho^{\prime}(r)+P_{0 s}(r) \rho(r)=0,
$$

where $s$ denotes the model employed in the present study. To make contact with Eq. (4.1), we differentiate Eq. (4.2) with respect to $r$ to find

$$
\begin{aligned}
& P_{2 s}(r) \rho^{\prime \prime \prime}(r)+\left[P_{1 s}(r)+P_{2 s}^{\prime}(r)\right] \rho^{\prime \prime}(r)+\left[P_{0 s}(r)+P_{1 s}^{\prime}(r)\right] \rho^{\prime}(r) \\
& \quad+P_{0 s}^{\prime}(r) \rho(r)=0 .
\end{aligned}
$$

The first point to emphasize is then the considerable similarity in "shape" between Eqs. (4.1) and (4.3). In particular, both are third-order, linear homogeneous differential equations for the ground-state density $\rho(r)$. Furthermore, in each case the density derivatives are multiplied by low-order polynomials. While it seems natural in making further comparison to neglect order- $(1 / Z)$ "corrections" in the polynomials entering Eq. (4.3), to compare with Eq. (4.1), it must be borne in mind that higher values of $l$, the orbital angular momentum quantum number, have been dropped from the one-center-like expansion of $e^{2} / r_{12}$ in reaching Eq. (4.3).

In this latter context, we recall the work of HoffmannOstenhof and Hoffmann-Ostenhof [5] which writes the asymptotic form of $\rho(r)$ at large $r$ as

$$
\rho(r) \rightarrow A r^{\alpha} \exp (-2 \sqrt{2 I} r),
$$

where $I$ is the ionization potential in units of $e^{2} / a_{0}$, with $r$ in units of the Bohr radius $a_{0}=\hbar^{2} / m e^{2}$. Evidently in the present He-like $s$-wave model, it follows from Eq. (2.8) that

$$
I(Z)=\frac{(Z-1)^{2}}{2}
$$

and that $\alpha=0$. March and Pucci [6] have suggested that in the Schwartz limit $\alpha$ has a small negative value proportional to $1 / Z$, and this is evidently then a detail of some significance which is not captured by the $s$-wave model underlying Eq. (4.3).

In concluding this section, it may be worthy of note that the density in Eq. (2.16) for the He $s$-wave model has two exponentials with exponents characterized by the ionization potential $I(Z)$ in Eq. (4.5), and this may be helpful in representing the density in the "true" He atomic ion sequence, though now presumably approximately. In the same context, $\alpha$ in the asymptotic large- $r$ form (4.4) may perhaps also depend, albeit weakly, on this ionization potential. It seems worthy of note that the x-ray scattering factor $f(k)$, the Fourier transform of $\rho(r)$, can also be characterized by $I(Z)$ when we use the explicit form (2.8), as shown in the Appendix.

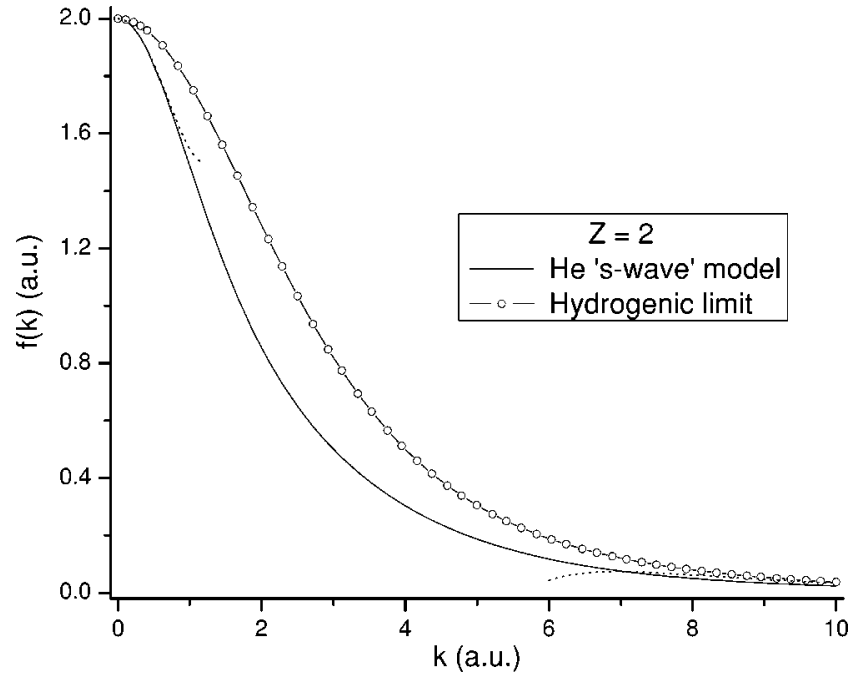

FIG. 3. X-ray scattering factor $f(k)$ derived from the density shown in Fig. 1 for $Z=2$, using the Fourier transform relationship (A1). The results of Eqs. (A2) and (A3) for small and large $k$, respectively, are indicated by the dashed lines.

\section{SUMMARY AND FUTURE DIRECTIONS}

The major results of the present study, based on the model Hamiltonian (2.1), are

(i) the exact result (2.15) for the nonrelativistic groundstate electron density for arbitrary atomic number $Z$ for the He-like atomic ions sequence;

(ii) the recognition that, for all $r$, the exponential terms in Eq. (2.15) are characterized by the ionization potential $I(Z)$ given in Eq. (4.5); and

(iii) the considerable similarity of shape between the new

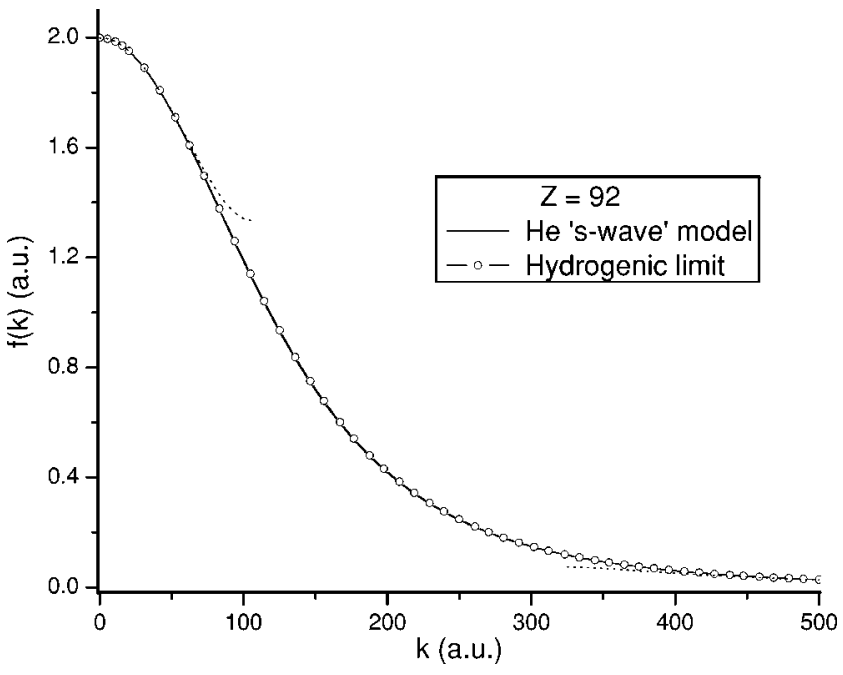

FIG. 4. X-ray scattering factor $f(k)$ derived from the density shown in Fig. 2 for $Z=92$, using Eq. (A1). Results of Eqs. (A2) and (A3) for small and large $k$, respectively, are indicated by the dashed lines. 
differential equation for the ground-state density derived here for the model Hamiltonian (2.1) and that satisfied by the Schwartz limit of large $Z$ recorded in Eq. (4.1). The present studies suggest that it should be useful for the future to seek a third-order, linear homogeneous differential equation for the nonrelativistic ground-state density of the "true" He-like atomic ions series. This, we suggest, may, at least approximately, lead to a density $\rho(r)$ which, for all $r$, may be characterized by the (exact) ionization potential $I(Z)$. The way $\alpha$ in Eq. (4.4) also involves $I(Z)$, but now as a "correction," seems to be a further direction worth pursuing. In that context, it will be essential to include the $p$ term in the expansion of $e^{2} / r_{12}$ at least-namely, $-2 r_{2} \cos \theta_{12} / r_{1}^{2}$ if $r_{1}>r_{2}$, etc. $<>$ But new methods of solution must then be devised.

\section{APPENDIX: X-RAY SCATTERING FACTOR CORRESPONDING TO ELECTRON DENSITY IN Eq. (2.11)}

The x-ray scattering factor $f(k)$ is defined as the Fourier transform of the electron density $\rho(r)$ which, with spherical symmetry, yields

$$
f(k)=\int \rho(r) \exp (i \mathbf{k} \cdot \mathbf{r}) d \mathbf{r}=4 \pi \int_{0}^{\infty} \rho(r) \frac{\sin (k r)}{k r} r^{2} d r .
$$

Inserting Eq. (2.11) into Eq. (A1), the integration can be performed analytically, but as the detail is considerable we shall merely plot $f(k)$ versus $k$ in Figs. 3 and 4 for $Z=2$ and $Z=92$. A series expansion of $f(k)$ gives

$$
\begin{aligned}
f(k) \simeq & 2-\frac{\left(128 Z^{4}-401 Z^{3}+484 Z^{2}-266 Z+56\right)}{2\left(64 Z^{6}-292 Z^{5}+548 Z^{4}-541 Z^{3}+296 Z^{2}-85 Z+10\right)} k^{2} \\
& +\frac{3}{16} \frac{\left(512 Z^{6}-2236 Z^{5}+4200 Z^{4}-4342 Z^{3}+2602 Z^{2}-855 Z+120\right)}{\left(256 Z^{10}-1936 Z^{9}+6528 Z^{8}-12920 Z^{7}+16616 Z^{6}-14505 Z^{5}+8702 Z^{4}-3542 Z^{3}+936 Z^{2}-145 Z+10\right)} k^{4}+O\left(k^{6}\right)
\end{aligned}
$$

for small $k$. For $k$ large, we have

$$
\begin{aligned}
f(k) \rightarrow & \frac{16 Z\left(32 Z^{5}-80 Z^{4}+80 Z^{3}-40 Z^{2}+10 Z-1\right)}{\left(16 Z^{2}-25 Z+10\right) k^{4}} \\
& +O\left(1 / k^{6}\right) .
\end{aligned}
$$

For comparison, the scattering factor $f_{H}(k)$ for the hydrogenic case is

$$
f_{H}(k)=\frac{32 Z^{4}}{\left(4 Z^{2}+k^{2}\right)^{2}},
$$

with the limiting forms

$$
f_{H}(k) \simeq 2-\left(\frac{k}{Z}\right)^{2}+\frac{3}{8}\left(\frac{k}{Z}\right)^{4}+O\left(k^{6}\right)
$$

for small $k$ and

$$
f_{H}(k) \rightarrow 32\left(\frac{Z}{k}\right)^{4}-256\left(\frac{Z}{k}\right)^{6}+O\left(1 / k^{8}\right)
$$

for large $k$.

\section{ACKNOWLEDGMENTS}

I.A.H. acknowledges support from the IWT-Flemish region under Grant No. IWT-161. N.H.M. wishes to thank Dr. G.E. Kilby for early collaboration on a related model of the He Hamiltonian, which undoubtedly had an important influence on the present study.
[1] G. Handke, Phys. Rev. A 50, R3561 (1994).

[2] T. Gál, N. H. March, and Á. Nagy, Chem. Phys. Lett. 305, 429 (1999).

[3] T. Kato, Commun. Pure Appl. Math. 10, 151 (1957).
[4] C. Schwartz, Ann. Phys. (N.Y.) 6, 156 (1959).

[5] M. Hoffmann-Ostenhof and T. Hoffmann-Ostenhof, Phys. Rev. A 16, 1782 (1977).

[6] N. H. March and R. Pucci, Phys. Lett. 85A, 75 (1981). 